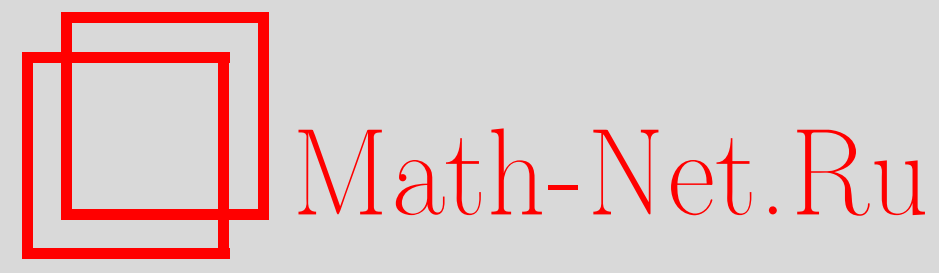

П. М. Ахметьев, Д. Реповш, М. Ценцель, О некоторых алгебраических свойствах диаграмм Серфа однопараметрических семейств функций, Функи. анализ и его прил., 2005, том 39, выпуск 3, 1-13

DOI: https://doi.org/10.4213/faa70

Использование Общероссийского математического портала Math-Net.Ru подразумевает, что вы прочитали и согласны с пользовательским соглашением

http://www.mathnet.ru/rus/agreement

Параметры загрузки:

IP: 54.162 .127 .20

26 апреля 2023 г., 04:37:43

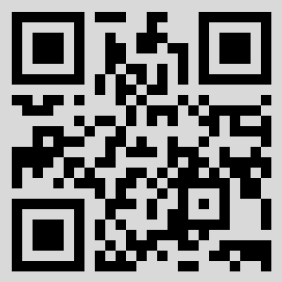


Функииональный анализ и его приложения

2005, т. 39, вып. 3, с. 1-13

УДК 515.142

\title{
О некоторых алгебраических свойствах диаграмм Серфа однопараметрических семейств функций*
}

\author{
(C) 2005. П. М. Ахметьев, Д. РЕПОВш, М. ЦЕНЦЕЛЬ
}

Диаграммой Серфа семейства функций $f_{\lambda}, \lambda \in[0,1]^{k}$, на многообразии называется гиперповерхность с особенностями в $[0,1]^{k} \times \mathbb{R}$, состоящая из всевозможных пар точек $(\lambda, x)$, где $x$ - одно из критических значений функции $f_{\lambda}$. В работе [3] были получены некоторые топологические ограничения на глобальную структуру диаграмм Серфа двупараметрического семейства, которые имеют правильные локальные особенности. В настоящей работе мы доказываем аналогичный результат для диаграмм Серфа однопараметрического семейства функций.

\section{§1. Введение}

Начальной точкой исследования послужила работа В. И. Арнольда [1] (см. также [2, задача 1988-23], где приводится ссылка на более раннюю работу), в которой сформулирована проблема переноса конструкции Понтрягина-Тома $[16,19]$ на новый класс задач, связанный с пространствами функций.

Параллельно с работами В. И. Арнольда появились работы, где конструкция Понтрягина-Тома была перенесена из категории кобордизмов многообразий в категорию кобордизмов отображений с особенностями. Идея такого обобщения впервые возникла в работе Сюча [18]; далее она была разработана и для отображений с более сложными особенностями в совместной работе [17] этого же автора с Римани. Недавно конструкция Понтрягина-Тома нашла применение при решении задач аппроксимации отображений с особенностями гладкими вложениями [5].

В работе [1] было замечено, что вычисление фундаментальной группы пространства вещественнозначных функций на прямой, принимающих постоянные значения в окрестности бесконечности и не имеющих особенностей типа $A_{3}$ и более сложных, эквивалентно вычислению группы кобордизмов плоских кривых, не имеющих точек горизонтального перегиба. Было доказано, что эта группа изоморфна группе целых чисел по сложению. Решение задачи 1988-23 предполагает перенесение этих вычислений на пространство функций на многообразии высокой размерности. Такое исследование было предпринято первым автором в работе [3], где конструкция Понтрягина-Тома применяется в форме Уэллса [20]. Рассмотрение было проведено лишь для первых двух гомотопических

* Работа первого автора выполнена при частичной поддержке Российского фонда фундаментальных исследований (проекты 03-05-64656 и 05-01-00993) и гранта INTAS N00-0259; работа второго и третьего авторов выполнена при частичной поддержке Министерства образования, науки и спорта Республики Словении, грант №0101-509. 
групп (т. е. для случая, когда параметров в задаче не больше 2); при этом высшие особенности на пространстве функций, за исключением морсовских особенностей и особенностей типа рождения-гибели пары морсовских особых точек, запрещены.

Недавно авторам стало известно, что теорема запрета из [3], доказательство которой весьма сложно, может быть получена более простым способом как следствие теоремы Игусы-Лауденбаха [11-13], которая цитируется ниже, и $h$-принципа. Применительно к теории псевдоизотопий $h$-принцип развивался Элиашбергом и Мишачевым в серии работ $[7,8]$. Обстоятельный обзор основных результатов по $h$-принципу имеется в книге [9]. Мы собираемся впоследствии передоказать (и обобщить) теорему из [3] в рамках указанного метода.

В настоящей работе мы рассматриваем аналог теоремы запрета. В одномерном случае запретов на диаграмму Серфа не возникает, но возникает важное, на наш взгляд, понятие ложного ринкла, или перекрученного ринкла, если использовать термин «ринкл» в смысле [7]. Имеется ввиду компонента кривой особых точек, образ которой в пространстве значений и параметров представлен простейшей диаграммой Серфа (линзой) с разными коэффициентами инцидентности сепаратрисных дисков в точках рождения-гибели. Она изображена ниже на рис. $1 \beta$ (см. с. 6). Нечетные количества таких ложных ринклов не могут образовывать диаграмму Серфа.

Диаграмма Серфа однопараметрического семейства функций на многообразии $M \times I$ представляет собой кривую с остриями, которая расположена с самопересечениями в пространстве значений и параметров. В остриях диаграммы происходит рождение или гибель пары морсовских критических точек соседних индексов. Предположим, что стабильные сепаратрисные диски морсовских критических точек снабжены согласованным семейством ориентаций. В остриях диаграммы определена дополнительная структура, связанная с коэффициентом пересечения стабильного и нестабильного сепаратрисных дисков, рождающихся или погибающих в окрестности точки рождения или гибели.

Для того чтобы определить эту дополнительную структуру, рассмотрим произвольную сепаратрису, связывающую две морсовские точки соседних индексов $i+1, i$. Такая сепаратриса представляет собой кривую, лежащую в пересечении стабильного сепаратрисного диска точки индекса $i+1$ и нестабильного сепаратрисного диска точки индекса $i$ в предположении, что эти диски пересекаются трансверсально. Рассмотрим произвольную ориентацию многообразия $M \times I$ в окрестности морсовской точки $x$ индекса $i$. Выбранная ориентация позволяет определить ориентацию нестабильного сепаратрисного диска в рассматриваемой критической точке так, что ориентация многообразия в окрестности точки $x$ получается как сумма ориентаций двух сепаратрисных дисков в этой точке: стабильного, на котором ориентация задана по условию, и нестабильного.

Выбор указанных ориентаций определяет коэффициент \pm 1 инцидентности сепаратрисных дисков. Легко проверить, что коэффициент не меняется, если ориентацию многообразия в точке $x$ изменить на противоположную. Если провести эти рассуждения для пары критических точек в окрестности точки рождения или гибели, то соответствующему острию на диаграмме Серфа удается 
приписать знак \pm 1 . Выбор знака в острие зависит от выбора семейства ориентаций стабильных сепаратрисных дисков и, следовательно, неоднозначен. Он производится с точностью до одновременного изменения коэффициентов в произвольной паре остриев, которая соединена между собой общим отрезком морсовских критических значений на диаграмме. Поэтому каждую диаграмму Серфа можно охарактеризовать как нескрученную или скрученную в зависимости от четности (0 или $1(\bmod 2)$ соответственно) числа остриев, имеющих одинаковые коэффициенты. При этом заметим, что ринклам соответствуют нескрученные диаграммы.

Оказывается, что диаграмма Серфа является скрученной, в точности если она имеет нечетное число точек трансверсального самопересечения. Это доказано в теореме из $§ 3$. Например, диаграмма на рис. $1 \gamma$ скрученная, поскольку самопересекается в одной точке.

Мы приводим два независимых доказательства этой теоремы. Одно из доказательств использует методы $K$-теории. Наши построения просты, так как высшие этажи $K$-теории не рассматриваются, рассматривается лишь функтор $K_{1}$, т. е. определитель. Использование этого функтора доставляет нетривиальное алгебраическое соотношение, которому подчиняются диаграммы Серфа функционального семейства, представляющего элемент из $\pi_{1}(P(M), E(M))$. Такой подход мотивирован визуализацией высшего алгебраического $K$-функтора, свойства которого к настоящему времени для целочисленных групповых колец фундаментальной группы изучены не в полной мере (см. [4] по поводу возможных топологических приложений).

Структура работы такова: в 2 мы напоминаем (в минимальной общности) основные определения, которые фактически были объяснены выше. Далее, в §3 формулируется основная теорема, которая доказывается в $\S 4$ с позиций $K$-теории, а в 5 методом Мишачева-Элиашберга.

\section{§2. Основные пространства}

Пусть $M$ - замкнутое многообразие и $I=[0,1]$. Пространство псевдоизотопий $E(M \times I)$ изучалось в работе Серфа [6] (мы сохраняем обозначение, принятое в этой работе). Оно было определено там как пространство функций $f: M \times I \rightarrow I, f(M \times\{0\})=0, f(M \times\{1\})=1$, таких, что $f$ не имеет критических точек и совпадает в некоторой окрестности границы (размеры которой не фиксируются) со стандартной проекцией на второй сомножитель. Это пространство имеет гомотопический тип пространства автодиффеоморфизмов многообразия $M \times I$, тождественных на нижнем основании.

Формальным аналогом $h E(M \times I)$ пространства $E(M \times I)$ служит пространство ненулевых сечений касательного расслоения $T(M \times I)$, совпадающих в некоторой окрестности границы с сечением по направлению I. Предполагается, что на $M$ задана риманова метрика. Тогда определено отображение $h_{E}: E(M \times I) \rightarrow h E(M \times I)$, сопоставляющее функции $f$ ее градиентное поле. Если $M$ односвязно, то по теореме Серфа $E(M \times I)$ связно. Отображение $h_{E}$ (при любом $M$ ) гомотопно отображению в точку. Этот результат называется в [7] теоремой Игусы-Лауденбаха. 
Для изучения гомотопического типа пространства $E(M \times I)$ определяется пространство $P(M \times I)$ функций с обобщенными морсовскими особенностями, т. е. морсовскими особенностями и особенностями типа рождения-гибели пары морсовских особых точек. Это пространство и его формальный аналог $h P(M \times I)$ мы определяем в следующем параграфе. В работе [7] доказано, что естественное включение $E(M \times I) \subset P(M \times I)$ является стягиваемым. Впрочем, первоначально близкий результат о стягиваемости этого отображения вплоть до некоторой размерности был доказан Игусой в [12]. В работе [8] доказано, что пространство $P(M \times I)$ слабо гомотопически эквивалентно своему формальному аналогу $h P(M \times I)$. Доказательство весьма сложно и использует специальную технику, разработанную в [7].

Рассмотрим теперь многообразие $N$ размерности $m+1$ с, вообще говоря, непустой границей. Обозначим через $F(N)$ пространство всех гладких вещественнозначных функций, определенных на данном многообразии и совпадающих в окрестности границы с заданной функцией $\varphi: N \rightarrow \mathbb{R}^{1}$, которая в этой окрестности регулярна.

Пусть $\Re \subset J^{k}(N)$ - открытое подпространство в многообразии струй, инвариантное относительно левых замен координат и, вдобавок, дополнительное к полуалгебраическому множеству. Многообразие струй снабжено расслоением $\kappa: J^{k}(N) \rightarrow N$. Определим пространство $A(N, \Re, \varphi)$ функций с $\Re$-умеренными особенностями как подпространство в $F(N)$, состоящее из функций, струи которых лежат в $\Re$, причем в сколь угодно малой окрестности границы $\partial N$ каждая такая функция совпадает с заданной регулярной функцией $\varphi$. Если $N=M \times I$ и $\Re$ совпадает со всем пространством $J^{k}(M \times I)$, то пространство $A(M \times I, \Re, p)$ совпадает с рассмотренным выше пространством $F(M \times I)$.

Формальным аналогом пространства функций $A(N, \Re, \varphi)$ для каждого фиксированного значения $k$ служит пространство $h A(N, \Re, \varphi)$ сечений расслоения $\kappa$, значения которых лежат в подпространстве $\Re$ и которые имеют в сколь угодно малой окрестности границы $k$-струю, совпадающую с $k$-струей заданного регулярного отображения. В случае $N=\mathbb{S}^{m} \times I$ можно определить формальный аналог $h A$ как подпространство в пространстве отображений Мар $\left[\mathbb{S}^{m} \times I ; \Re\right]$ со значениями в $\Re$ и заданными граничными условиями.

Определено отображение $A(N, \Re, \varphi) \rightarrow h A(N, \Re, \varphi)$, сопоставляющее гладкой функции ее $k$-струйное расширение.

Исходная задача вычисления гомотопического типа пространства $A$ может решаться при помощи методов алгебраической топологии путем вычисления гомотопического типа пространства $h A$. Такая редукция называется $h$-принципом. Во многих задачах формальный аналог $h A$ оказывается слабо гомотопически эквивалентным исходному пространству функций $A$; см. по этому поводу работы Васильева $[14,15]$. Из теоремы Мишачева-Элиашберга вытекает, что $h$-принцип справедлив для пространства функций, имеющих только особенности типов $A_{1}$ и $A_{2}$.

Определение пространства $\boldsymbol{P}(\boldsymbol{M} \times \mathbf{I})$ обобщенных морсовских функций. Скажем, что функция $f: M \times I \rightarrow I, f \in F(M \times I)$, является обобщенной функцией Морса, если при некотором $i$, значение которого может меняться от 0 
до $m$, ее особенности задаются следующими формулами в некоторой локальной системе координат:

$$
\begin{gathered}
-x_{1}^{2}-\cdots-x_{i}^{2}+x_{i+1}^{2}+\cdots+x_{m+1}^{2}, \\
-x_{1}^{2}-\cdots-x_{i}^{2}+x_{i+1}^{2}+\cdots+x_{m}^{2}+x_{m+1}^{3} .
\end{gathered}
$$

Точки типа $A_{1}$ называются морсовскими особыми точками индекса $i$. Точки типа $A_{2}$ называются точками рождения-гибели пары морсовских критических точек индексов $i$ и $i+1$.

Пространство $P(M \times I)$ функций с обобщенными морсовскими особенностями определено как подпространство в $F(M)$ функций с особенностями только типов $A_{1}$ и $A_{2}$. Согласно предыдущим конструкциям, определен формальный аналог $h A(M \times I, \Re, p)$, где $\Re$ - подпространство в пространстве струй без особенностей типа $A_{3}$ и более сложных, а $p$ - стандартная проекция на второй сомножитель. Для краткости пространство $h A(M \times I, \Re, p)$ мы будем обозначать через $h P(M)$.

Пространство $\boldsymbol{h o l} \boldsymbol{P}(\boldsymbol{M} \times \boldsymbol{I})$. В пространстве $h P(M \times I)$ формальных функций определим подпространство $h o l P(M \times I)$, состоящее из формальных функций $h f: M \times I_{x} \rightarrow J^{3}(M \times I, \mathbb{R})$, удовлетворяющих следующим дополнительным условиям: существует открытое подмножество $U \subset M \times I_{x}$, такое, что $\left.h f\right|_{M \times I \backslash U}$ не имеет критических точек и $\left.h f\right|_{U}$ совпадает со струйным расширением некоторой функции $f: M \times I_{x} \rightarrow I_{y}$ из $P(M)$. Иными словами, формальная функция $h f: M \times I_{x} \rightarrow I_{y}$ должна быть голономной в окрестности своих критических точек. Это пространство включено в диаграмму

$$
P \stackrel{i_{h o l}}{\complement} h o l P \stackrel{i_{h}}{\complement} h P .
$$

Пространство $E(M \times I)$ и его формальный аналог $h E(M \times I)$ в случае стабильно параллелизуемого многообразия $\boldsymbol{M}$. Если $M$ риманово и $k=1$, то пространство $h E(M \times I)$ естественно отождествляется с пространством ненулевых сечений касательного расслоения $T(M \times I)$ со стандартными условиями на границе. В случае когда $M$ стабильно параллелизуемо, расслоение $T(M \times I)$ тривиально и пространство $h E(M \times I)$ можно описать как пространство отображений $M \times I \rightarrow \mathbb{S}^{m}$, которые переводят границу многообразия $M \times\{0\} \cup M \times\{1\}$ в отмеченную точку на $\mathbb{S}^{m}$. Определено отображение забывания $h_{E}: E(M \times I) \rightarrow h E(M \times I)$. По теореме Игусы-Лауденбаха это отображение стягиваемо. (Это отображение не является взаимно однозначным на компонентах связности при неодносвязном $M$, так как пространство $E(M \times I)$ оказывается в этом случае несвязным, в то время как пространство $h E(M \times I)$ связно.) Компоненты связности пространства $h E(M \times I)$ описываются в [10]. Несмотря на то, что пространства $E(M \times I), h E(M \times I)$ оказались гомотопически различными, Мишачеву и Элиашбергу (см. [7]) удалось построить функциональное пространство, формальным аналогом которого служит пространство $h E(M \times I)$. Это пространство функций, имеющих лишь морсовские и обобщенные морсовские критические точки, с некоторой дополнительной структурой, задающей систему координат в некотором диске, который содержит пару критических точек соседнего индекса или точку рождения или гибели. Диаграммы 
Серфа для функций из этого пространства имеют простейший вид и называются ринклами. Стандартный ринкл для однопараметрического семейства функций изображен на рис. $1 \alpha$.

Построенные выше пространства включены в коммутативную диаграмму

$$
\begin{aligned}
& \begin{array}{lll}
P(M \times I) & \supset & E(M \times I)
\end{array} \\
& h_{P} \downarrow \\
& \downarrow h_{E} \\
& h(P(M \times I)) \supset \operatorname{hol} P(M \times I) \supset h E(M \times I)
\end{aligned}
$$

\section{§3. Формулировка основного результата}

Напомним, что представитель элемента группы $\pi_{1}(P)$ или, что эквивалентно, группы $\pi_{0}(\Omega P)$, может быть изучен при помощи диаграммы Серфа; см. $[6,7]$. Рассмотрим диаграммы Серфа $\alpha, \beta, \gamma, \delta$, изображенные на рис. 1.

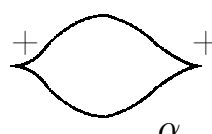

$\alpha$

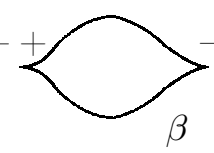

$\beta$

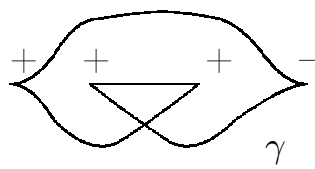

Рис. 1

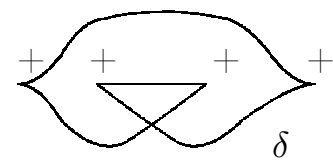

Легко построить пример семейств функций $f_{\lambda}, g_{\lambda}, \lambda \in[0,1]$, с диаграммами $\alpha$ (ринкл) и $\gamma$ соответственно, снабженных семейством ориентаций $O(\lambda)$ (см., например, [6]). Каждое из семейств, представленных на этом рисунке, имеет лишь критические точки двух соседних индексов, $i+1, i$. Критические точки этих семейств мы будем далее обозначать через $x$ с нижним индексом.

Зафиксируем некоторое семейство ориентаций $O(\lambda)$ на множестве стабильных сепаратрисных дисков критических морсовских точек каждого из семейств $\alpha-\delta$. При этом предполагается, что ориентация меняется непрерывно с изменением параметра семейства.

Рассмотрим семейства $O(\lambda)$ в окрестности точек рождения-гибели. Каждая критическая точка $x$ этого типа (скажем, любая особенность типа рождениягибели на диаграмме $1 \alpha$ ) снабжена знаком $o(x)= \pm 1$ в соответствии со знаком коэффициента инцидентности пары морсовских критически точек, рождающихся или погибающих в этой особой точке. Очевидно, что для семейства $\alpha$ выполнено соотношение

$$
o\left(x_{1}\right) o\left(x_{2}\right)=+1,
$$

в то время как для семейства $\gamma$ выполнено соотношение

$$
o\left(x_{1}\right) o\left(x_{2}\right) o\left(x_{3}\right) o\left(x_{4}\right)=-1 .
$$

Диаграммы $\beta$ (ложный ринкл) и $\delta$ также можно рассматривать с формальной точки зрения как диаграммы Серфа для семейства функций пространства hol $P(M)$. Для диаграммы $\beta$ имеем

$$
o\left(x_{1}\right) o\left(x_{2}\right)=-1,
$$

а для диаграммы $\delta$

$$
o\left(x_{1}\right) o\left(x_{2}\right) o\left(x_{3}\right) o\left(x_{4}\right)=+1 .
$$


Диаграммы Серфа можно определить и для формальных семейств hol $\alpha$, $h o l \beta$, которые моделируют соответствующие семейства в группе $\pi_{1}(h o l P)$. Ecли зафиксировать семейства ориентаций $h O(\lambda)$ на стабильных сепаратрисных дисках критических точек формальных семейств, то для каждой критической точки $h x$ рождения-гибели также определен знак $o(h x)= \pm 1$. Поскольку семейства функций пространства hol $P$ голономны в окрестности своих критических точек, то для таких семейств можно говорить о диаграммах Серфа.

Рассмотрим элемент $\phi$ гомотопической группы $\pi_{1}(P, E)$ и диаграмму Серфа, представляющую этот элемент. Выберем дополнительную структуру $O(\lambda)$ ориентаций сепаратрисных многообразий. Без учета дополнительной структуры $O$ диаграмма Серфа представлена кривой $S$ критических значений в двумерном пространстве значений и параметров. Кривая $S$ имеет особенности в точках множества $\Sigma$ критических значений типа рождения-гибели. После сглаживания кривой $S$ вдоль этого особого множества $\Sigma$ получим погруженное подмногообразие, которое мы будем снова обозначать через $S$. Согласно конструкции Понтрягина-Тома в форме Уэллса $[16,19,20]$, многообразие $S$ представляет элемент $\kappa(\phi)$ в стабильной гомотопической группе сфер $\pi_{n+1}\left(\mathbb{S}^{n}\right), n>2$, которая обозначается через $\Pi_{1}$. Класс кобордизма этого элемента полностью определяется четностью числа точек самопересечения погруженной кривой $S$ в квадрате значений и параметров $I^{2}$.

На нульмерном множестве $\Sigma$ критических значений типа рождения-гибели дополнительная структура $O$, описанная в начале настоящего параграфа, позволяет определить его каноническое разбиение на два подмножества (нульмерных многообразия), $\Sigma=\Sigma_{+} \cup \Sigma_{-}$, в соответствии со значением коэффициента инцидентности пары морсовских особенностей в малой окрестности компоненты критических точек. Класс кобордизма любого из многообразий $\Sigma_{+}$или $\Sigma_{-}$ (многообразия $\Sigma_{+}$и $\Sigma_{-}$кобордантны как погруженные многообразия) определяет элемент $\rho(\phi)$ в стабильной гомотопической группе сфер $\Pi_{0}=\pi_{n}\left(\mathbb{S}^{n}\right)$, $n \geqslant 1$. Этот элемент, вообще говоря, зависит от выбора структуры $O$ на многообразии сепаратрисных дисков. Произвол в выборе структуры $O$ не влияет на элемент $\rho(\phi)$, если рассматривать этот элемент в факторгруппе $\Pi_{0} / 2 \Pi_{0}$. Действительно, каждый отрезок, состоящий из морсовских особенностей, на диаграмме $S$ ограничен парой особенностей из $\Sigma$. Смена ориентации на многообразии стабильных сепаратрисных дисков вдоль этого отрезка изменяет типы особенностей из $\Sigma$ на его концах. Следовательно, элемент $\rho(\phi)(\bmod 4)$ не зависит от выбора структуры $O$. С другой стороны, при кобордизме особенности из $\Sigma$ уничтожаются парами. При согласованном выборе структуры $O$ на кривой особых точек до перестройки (после перестройки) в окрестности точки исчезновения (появления) пары критических точек рождения-гибели знаки в соответствующей паре остриев кривой $S$ противоположны и структура $O$ продолжается на кобордизм. Значит, элемент в факторгруппе $\Pi_{0} / 2 \Pi_{0}$ определяет инвариант на классе кобордизма диаграммы Серфа.

Обозначим через $t$ образующую в $\Pi_{1}$, которая представлена гомотопическим классом надстройки над отображением Хопфа $\mathbb{S}^{3} \rightarrow \mathbb{S}^{2}$; в терминах конструкции Понтрягина-Тома эта образующая представляется классом кобордизма плоской погруженной кривой формы горизонтальной восьмерки. 
Теорема. Справедливо равенство

$$
t \circ \rho(\phi)=\kappa(\phi),
$$

в котором левая часть обозначает композииию стабильных сфероидов.

ПримеР. Для пути в пространстве функций с диаграммой Серфа, изображенной на рис. $1 \alpha$ или $1 \delta$, выполнено равенство $\kappa=t$. Для семейств на рис. $1 \beta$ или $1 \gamma$ элемент $\rho$ является элементом нечетного порядка в группе $\Pi_{1}$.

\section{§4. Доказательство теоремы при помощи функтора $K_{1}$}

В этом параграфе предполагается, что $\operatorname{dim}(M)=m \geqslant 5$. Пользуясь параметрической версией леммы Смейла (см. [10]), легко доказать, что в гомотопическом классе $\phi \in \pi_{1}(P(M), E(M))$ найдется такой путь $f_{\lambda}, \lambda \in[0,1]$, что выполнены следующие условия:

1) семейство функций $f_{\lambda}$ имеет лишь критические точки индексов $i+1, i$, где $i=[m / 2]$;

2 ) критические значения индекса $i+1$ (соответственно $i$ ) лежат в интервале $(1 / 2,1)$ (соответственно $(0,1 / 2))$.

При замене пути $f_{\lambda}$ на гомотопный диаграмма Серфа меняется на кобордантную. Очевидно, что при этом обе части равенства (4) сохраняются, так как особенности типа $A_{3}$ «ласточкин хвост» при кобордизме не появляются. Докажем формулу (4), предполагая, что элемент $\phi$ представлен семейством функций $f_{\lambda}$, удовлетворяющих условиям 1$\left.), 2\right)$. Для произвольного значения $\lambda \in[0,1]$, за исключением конечного множества особых значений, определена целочисленная матрица коэффициентов инцидентности $A(\lambda)$ (здесь выбрана структура $O(\lambda))$ критических точек в комплексе Морса многообразия $M \times I$, порожденном функцией $f_{\lambda}$. Критические точки индекса $i+1$ (соответственно $i$ ) нумеруются, начиная с 1 , и их порядок определяется в соответствии с убыванием (возрастанием) критических значений. Условимся, что матрица $A(\lambda)$ стабилизирована элементом +1 в клетке $(0,0)$; это потребуется в случае, когда критических точек нет. Рассмотрим особые значения параметра $\lambda$, которые классифицируются следующим списком особенностей.

а. Особенности типа рождения-гибели пары критических морсовских точек соседних индексов. Заметим, что дополнительным преобразованием стабильного сепаратрисного диска для точки индекса $i+1$ и нестабильного сепаратрисного диска для точки индекса $i$ в окрестности точки рождения или гибели можно добиться того, чтобы множество особых сепаратрисных траекторий, лежащих на этих дисках, содержало единственную траекторию, которая соединяла бы эти две критические точки с коэффициентом инцидентности \pm 1 (при определении этого коэффициента предполагается, что выбрана структура $O$ ). Тогда знак этой траектории, т. е. коэффициент инцидентности рассматриваемой пары особых точек, позволяет определить разбиение множества $\Sigma$ на подмножества $\Sigma_{+}, \Sigma_{-}$.

b. Особенности типа прибавления-вычитания ручки в клеточном разбиении, порожденном функцией Морса.

с. Особенность типа совпадения двух критических значений (в соответствии с правилом 2 , значений одного индекса). 
Теперь рассмотрим те особенности, которые соответствуют точкам разрыва функции $\operatorname{det}(A(\lambda))$, принимающей значения \pm 1 . Разрыв этой функции обусловлен двумя причинами: совпадением пары критических значений (что приводит к умножению матрицы $A$ слева или справа на матрицу элементарной транспозиции в зависимости от индекса совпадающих критических значений) или наличием точки рождения-гибели из $\Sigma_{-}$, что приводит к отщеплению одномерной скалярной матрицы -1 от правого нижнего диагонального элемента. Число точек разрыва функции $\operatorname{det}(A(\lambda))$ четно, так как при $\lambda=0, \lambda=1$ функция $f_{\lambda}$ не имеет критических точек и $A(\lambda)$ принимает значения +1 . Следовательно, четность числа $x$ точек самопересечения на диаграмме Серфа совпадает с четностью числа $y$ особенностей из $\Sigma_{-}$. Теперь заметим, что значение гомоморфизма $\kappa$, построенного по этой диаграмме, определяет образующую в П 1 тогда и только тогда, когда $x=1(\bmod 2)$. Далее, значение $y(\bmod 2)$ определяет элемент в группе $\Pi_{0} / 2 \Pi_{0}$. Итак, $x=y(\bmod 2)$ или, эквивалентно, $\kappa=t \circ \rho$. Теорема доказана.

\section{§5. Доказательство теоремы при помощи $h$-принципа}

В рамках этого доказательства мы предполагаем, что многообразие $M$ является стабильно-параллелизованным и односвязным. Рассмотрим последовательность

$$
\pi_{1}(P(M), E(M)) \stackrel{\left(h_{P}, h_{E}\right)_{\sharp}}{\longrightarrow} \pi_{1}(h o l P(M), E(M)) \stackrel{\text { hol } \kappa \oplus h o l \rho}{\longrightarrow} \Pi_{1} \oplus \Pi_{1} .
$$

Гомоморфизм $h o l \kappa \oplus \operatorname{hol} \rho$ мы определяем на группе $\pi_{1}(h o l P(M), E(M))$ по аналогии с гомоморфизмом $\kappa \oplus \rho$ так, чтобы выполнялось равенство (hol $\kappa \oplus$ hol $\rho) \circ h_{P}=\kappa \oplus \rho$.

Вычислим образ гомоморфизма $\kappa \oplus \rho$ в группе $\Pi_{1} \oplus \Pi_{0} / 2 \Pi_{0}$. Пусть $h f_{\lambda}$, $\lambda \in[0,1],-$ исследуемое семейство формальных функций, голономное в малой регулярной окрестности своих критических точек, а также в окрестности границы $\partial(M \times I) \cup(M \times\{0\} \cup M \times\{1\})$. В частности, функции $h f_{0}, h f_{1}$ являются голономными и не имеют критических точек.

Перестроим диаграмму Серфа семейства $h f_{\lambda}$ до одной из простейших диаграмм, изображенных на рис. 1. Заметим при этом, что все априори возможные классы кобордизма диаграмм представлены на этом рисунке. Более того, кобордизм диаграммы можно, не ограничивая общности, продолжить до гомотопии некоторого голономного семейства функций в регулярной окрестности кривой особых точек. Далее заметим, что семейство формальных функций также можно продеформировать с сохранением условий голономности в окрестности кривой особых точек. Действительно, кобордизм диаграммы, при котором все точки рождения-гибели сохраняются, можно продолжить до гомотопии формального семейства, пользуясь стандартными соображениями.

Обозначим параметр гомотопии через $\tau$. Рассмотрим диск $D_{0}^{n+2} \subset M \times I \times I$ с центром в особой точке исчезновения пары точек рождения-гибели. При этом предположим, что диск $D_{\tau_{0}-\varepsilon}=D_{0}^{n+2} \cap M \times I \times\left\{\tau_{0}-\varepsilon\right\}$ целиком содержит регулярную окрестность двух ветвей $S_{1}, S_{2}$ критических точек семейств $h f_{\lambda}\left(\tau_{0}-\varepsilon\right)$, зеркально симметричных по $\lambda$, и особенности из $\Sigma$, по одной на каждой ветви. 
Диск $D_{\tau_{0}+\varepsilon}=D_{0}^{n+2} \cap M \times I \times\left\{\tau_{0}+\varepsilon\right\}$ содержит регулярную окрестность перестроенной ветви $S$ кривой особых точек семейства $h f_{\lambda}\left(\tau_{0}+\varepsilon\right)$ и не содержит особенностей из $\Sigma$.

Нетрудно проверить, что деформацию, заданную в окрестности кривой особых точек, можно продолжить до деформации всего формального семейства при значениях параметра $\tau$ из $\left[\tau_{0}-\varepsilon, \tau_{0}+\varepsilon\right]$. Действительно, соединим при $\tau=\tau_{0}-\varepsilon$ точки рождения и гибели, лежащие на ветвях $S_{1}, S_{2}$, малым отрезком $J$. Дополнительной деформацией формального семейства $h f_{\lambda}$ с носителем в $D_{0}$ на отрезке $\tau_{0}-\varepsilon \leqslant \tau \leqslant \tau_{0}-\varepsilon / 2$ добьемся того, чтобы поле градиентов формального семейства $h f_{\lambda}\left(\tau_{0}-\varepsilon / 2\right)$ совпало с полем градиентов стандартного голономного семейства внутри $D_{0}$.

После этого построения продолжение деформации формального семейства при $\tau_{0}-\varepsilon / 2 \leqslant \tau \leqslant \tau_{0}+\varepsilon$ внутри $D_{0}$ очевидно, а вне $D_{0}$ оно строится стандартным способом.

Таким образом, достаточно показать, что диаграммы $\beta, \gamma$, представленные на рис. 1 , не могут быть реализованы ни для какого формального семейства $h f_{\lambda}$. Поскольку отображение $h_{E}: E \rightarrow h E$ стягиваемо, функции $h f_{0}, h f_{1}$ как формальные гомотопны постоянной. Изучим возможность реализации диаграммы Серфа в виде ложного ринкла $\beta$ на рис. 1 . Сначала опишем семейство функций в окрестности ложного ринкла в аналитической форме.

Пусть $f_{\lambda}$ - семейство функций с правильными граничными условиями, для которого диаграмма Серфа представляет собой стандартный ринкл $\alpha$ на рис. 1. Пусть $I_{0} \subset I_{\lambda}$ - отрезок в пространстве параметров деформаций ростков и $a \in$ $M^{m} \times I(x)$ - точка на многообразии. Выберем деформацию семейства $f_{\lambda}$ так, чтобы она удовлетворяла следующему техническому условию: в некоторой фиксированной шаровой окрестности $U_{r}(a) \subset M^{m} \times I$ радиуса $r$ точки $a$ функция $f_{\lambda}$ не зависит от $\lambda \in I_{0}$ и задана стандартной квадратичной формой $q$ индекса $i+1$. Для простоты дальнейших обозначений положим $r=2$ и $I_{0}=[1 / 3,2 / 3]$.

Переопределим значения семейства ростков $f_{\lambda}$ внутри шаров $U_{1}(a)=U_{1}$ при указанных значениях параметра $\lambda$; при этом вне шара семейство $f_{\lambda}$ менять не будем. После переопределения получится семейство $g_{\lambda}$, определенное внутри $U_{1} \times[1 / 3,2 / 3]$, причем его ограничение на $U_{1} \times\{1 / 3,2 / 3\}$ совпадает с семейством $f_{\lambda}$.

Проведем вспомогательные построения. Обозначим через $\Delta$ двумерную плоскость, проходящую через точку $a-$ центр шара $U_{2}-$ и определенную координатами $x_{1}, x_{i+2}$. Заметим, что ограничения квадратичных частей функций $f_{\lambda}$ на плоскость $\Delta$ не являются знакоопределенными. Не теряя общности, можно считать, что все эти ограничения совпадают между собой и с $q$. Рассмотрим однопараметрическое семейство вращений $Z_{\lambda}: U_{1} \rightarrow U_{1}$ в плоскости $\Delta$ в положительном направлении, параметризованных точками $\lambda \in I_{0}$, такое, что $Z_{1 / 3}$ - тождественное преобразование, а $Z_{2 / 3}-$ поворот на угол $\pi$. Определим $g_{\lambda}(b), b \in U_{1}$, по формуле $g_{\lambda}(b)=q\left(Z_{\lambda}(b)\right)$. Пары ростков $\left(g_{2 / 3}(x), f_{2 / 3}(x)\right)$ и $\left(g_{1 / 3}(x), f_{1 / 3}(x)\right)$ совпадают для любой точки $x \in U_{1}$. Поэтому определено семейство функций $g_{\lambda}$ в окрестности ложного ринкла.

Обозначим через $V_{1} \subset V_{2} \subset M \times I \times I$ пару окрестностей построенного ложного ринкла в пространстве прообразов и параметра, являющуюся расшире- 
нием пары окрестностей $U_{1} \times I_{0} \subset U_{2} \times I_{0}$ дуги ложного ринкла над отрезком $a \times I_{0}$. Вычислим препятствие к решению задачи продолжения внутрь полнотория $V_{2} \backslash V_{1}$ формального семейства, совпадающего с голономным семейством $f_{\lambda}$ на его внешней границе $\partial V_{2}$ и с $g_{\lambda}$, на его внутренней границе $\partial V_{1}$.

Семейство $g_{\lambda}$ на $\partial V_{1}\left(\partial V_{2}\right)$ задано ненулевым сечением расслоения $T(M \times I)$ и определяется отображением $G: \partial U_{1} \times I_{0} \rightarrow \mathbb{S}^{m}\left(G: \partial U_{2} \times I_{0} \rightarrow \mathbb{S}^{m}\right)$. Аналогично, семейство $f_{\lambda}$ на $\partial V_{1}\left(\partial V_{2}\right)$ определяется отображением $F: \partial U_{1} \times I_{0} \rightarrow \mathbb{S}^{m}$ $\left(F: \partial U_{2} \times I_{0} \rightarrow \mathbb{S}^{m}\right)$.

Легко видеть, что на $\partial V_{1}, \partial V_{2}$ отображение $G$ получается из $F$ в результате композиции с семейством вращений сферы $\mathbb{S}^{m}$ вокруг окружности $\mathbb{S}^{1} \subset \mathbb{S}^{m}$ на углы от 0 до $2 \pi$, параметризованные координатой на отрезке $I_{0}$. Заметим, что на боковой части $\left(U_{1} \backslash U_{2}\right) \times\left\{\partial I_{0}\right\}$ кольца $K=U_{1} \times I_{0} \backslash U_{2} \times I_{0}$ отображения $F$ и $G$ совпадают. Задача продолжения отображения внутрь кольца $K$ имеет препятствие $o(F, G)$ в группе когомологий $H^{m+1}\left(\partial K ; \pi_{m+1}\left(\mathbb{S}^{m}\right)\right)$. Значение этого препятствия определяется значением образующей $a \in \pi_{1}(S O(m))$ при гомоморфизме Джеймса-Уайтхеда $J: \pi_{1}(S O(m)) \rightarrow \pi_{m+1}\left(\mathbb{S}^{m}\right)=\Pi_{1}$. Известно, что $[J(a)]=t$, где $t \in \Pi_{1}$ - образующая. Следовательно, рассматриваемая задача продолжения отображения внутрь кольца $K$ не имеет решения. Задача продолжения отображения внутрь полнотория $V_{2} \backslash V_{1}$ также не имеет решения по аналогичным причинам.

Теперь докажем, что задача продолжения отображения $G$ с $\partial V_{1}$ до семейства формальных функций $h g_{\lambda}$ на всем пространстве $M \times I \times I$ со стандартными условиями на границе пространства прообраза и параметра также не имеет решения. Для этого изучим произвол в продолжении семейства формальных функций $h g_{\lambda}$ на $\partial(M \times I \times I)$ до семейства формальных функций на $\partial V_{2}$. Диаграмма $S$ заключена в шар $\partial D_{0}$, и ограничение семейства формальных функций $h g_{\lambda}$ на $\partial D_{0}$ однозначно, с точностью до гомотопии, задано постоянным отображением. Действительно, многообразие $M^{m} \times I \times I \backslash D_{0}$ является стабильно-параллелизованным многообразием, осуществляющим кобордизм между $\partial D_{0}$ и внешней границей $M^{m} \times \partial(I \times I)$. Семейство формальных функций $h g_{\lambda}$ на этом многообразии не имеет критических точек и представлено отображением $M^{m} \times I \times I \backslash D_{0} \rightarrow \mathbb{S}^{m}$. Поскольку $D_{0} \backslash V_{2}$ также является стабильно-параллелизованным $(m+2)$-мерным многообразием, задача продолжения отображения $\partial\left(D_{0} \backslash V_{1}\right) \rightarrow \mathbb{S}^{m}$ с границы на все $D_{0} \backslash V_{1}$ имеет препятствие в $\pi_{m+1}\left(\mathbb{S}^{m}\right)$. С одной стороны, ограничения формальных семейств $h g_{\lambda}: \partial D_{0} \rightarrow$ $\mathbb{S}^{m}, h f_{\lambda}: \partial D_{0} \rightarrow \mathbb{S}^{m}$ гомотопны (гомотопны постоянному отображению). Здесь через $h f_{\lambda}$ обозначено формальное семейство, определенное голономным семейством $f_{\lambda}$. С другой стороны, $G: \partial V_{1} \rightarrow \mathbb{S}^{m}$, как мы доказали выше, отличается от $F: \partial V_{1} \rightarrow \mathbb{S}^{m}$ на образующую из $\pi_{m+1}\left(\mathbb{S}^{m}\right)$. Поскольку для $F$ задача продолжения с $\partial\left(D_{0} \backslash V_{1}\right)$ на $D_{0} \backslash V_{1}$ разрешима, то для $G$ эта задача не имеет решения. Итак, доказано, что ложный ринкл не реализуется диаграммой Серфа ни для какого семейства формальных функций. Случай диаграммы $\delta$ разбирается аналогично. Образ гомоморфизма $h \kappa \oplus h \rho$ вычислен, и теорема доказана. 


\section{§6. Обсуждения}

Мы изучили однопараметрические семейства функций с обобщенными морсовскими особенностями и показали, что инварианты $\kappa$ и $\rho$, построенные по диаграммам Серфа соответствующих семейств в стабильных гомотопических группах сфер, являются зависимыми даже для семейств формальных функций. $\mathrm{C}$ алгебраической точки зрения рассматриваемое соотношение связано с функтором $K_{1}(\mathbb{Z})$, т. е. определителем, а с гомотопической точки зрения соотношение между значениями $\kappa$ и $\rho$ выражается при помощи образующей группы $\Pi_{1}$.

При рассмотрении двупараметрических семейств функций аналогичное алгебраическое соотношение уже связано с функтором $K_{2}(\mathbb{Z})$. В работе первого автора [3] высказана гипотеза о том, что некоторые диаграммы Серфа семейств функций, имеющие допустимое локальное строение (и выступающие как диаграммы Серфа семейств формальных функций), запрещены для семейств голономных функций. При формулировке гипотезы предполагалось, что семейство функций без критических значений на границе пространства параметров является формальным. Сформулированную гипотезу предпочтительно переформулировать в связи с последними результатами из [8]. Целесообразно было бы рассмотреть следующую проблему:

ПроблемА. Доказать результат о нереализуемости диаграмм Серфа двупараметрических семейств функций из [3] в рамках $h$-принципа МишачеваЭлиашберга. Неформально говоря, показать, что $h$-принцип в теории псевдоизотопий «помнит» $K$-теорию на уровне пространств формальных функций с умеренными особенностями.

Авторы благодарят рецензента за внимательное прочтение рукописи и обнаружение ошибки в первоначальном варианте статьи.

Первый автор благодарит П. Е. Пушкаря за полезные замечания.

\section{ЛитератУРА}

1. Арнолъд В. И. Пространства функций с умеренными особенностями. Функц. анализ и его прил., 23, вып. 3, 1-10 (1989).

2. Арнольд В. И. Задачи Арнольда. Фазис, М., 2000.

3. Ахметьев П. М. Вложения компактов, стабильные гомотопические группы сфер и теория особенностей. УМН, 55 , вып. 3, 3-62 (2000).

4. Ахметъев П. М. $K_{2}$ для простейших целочисленных групповых колец и возможные топологические приложения. Матем. сб., 194, № 1, 23-30 (2003).

5. Akhmetiev P. M. Pontrjagin-Thom construction for approximation of mappings by embeddings. Topology Appl., 140, No. 2-3, 133-149 (2004).

6. Cerf J. La stratification naturelle des espaces des fonctions differentiables reelles et le theoreme de la pseudoisotopie. Publ. Math. IHES, 39, 5-173 (1970).

7. Eliashberg Y., Mishachev N. M. Wrinkling of smooth mappings and its applications. I. Invent. Math., 130, 345-369 (1997).

8. Eliashberg Y., Mishachev N. M. Wrinkling of smooth mappings. II. Wrinkling of embeddings and K. Igusa's theorem. Topology, 39, No. 4, 711-732 (2000).

9. Eliashberg Y., Mishachev N. M. Introduction to the $h$-principle. Grad. Stud. Math., Vol. 48, Amer. Math. Soc., Providence, RI, 2002.

10. Hatcher A., Wagoner J. Pseudo-isotopy of compact manifolds. Asterisque, No. 6, Soc. Math. France, Paris, 1973. 
11. Igusa $K$. On the homotopy type of the space of generalized Morse functions. Topology, 23, No. 2, 245-256 (1984).

12. Igusa K. Higher singularities are unnecessary. Ann. of Math., 119, No. 1, 1-58 (1984).

13. Laudenbach $F$. Formes différentielles de degré 1 fermées non singulières: classes d'homotopie de leurs noyaux. Comment. Math. Helv., 51, No. 3, 447-464 (1976).

14. Васильев B. A. Топология дополнений к дискриминантам. Фазис, М., 1997.

15. Василъев B. A. Топология пространств функций без сложных особенностей. Функц. анализ и его прил., 23, вып. 4, 24-36 (1989).

16. Понтрягин Л. С. Гладкие многообразия и их применения в теории гомотопий. 2-е изд., Наука, М., 1976.

17. Rimanyi R., Szucs A. Pontrjagin-Thom type construction for maps with singularities. Topology, 37, No. 6, 1177-1191 (1998).

18. Сюч A. Аналог пространства Тома для отображений с особенностью типа $\Sigma^{1}$. Матем. сб., 108 (150), №3, 438-456 (1979).

19. Thom $R$. Quelques propriétés globales des variétés différentiables. Comment. Math. Helv., 28, 17-86 (1954). [Имеется перевод: Том P. Некоторые свойства в целом дифференцируемых многообразий. В кн.: Расслоенные пространства. ИЛ, М., 1958, c. $291-351$.

20. Wells R. Cobordism groups of immersions. Topology, 5, 281-294 (1966).

ИЗМИРАН, Троицк Московской обл.

e-mail: pmakhmet@mi.ras.ru

Institute for Mathematics, Physics and Mechanics,

University of Ljubljana

e-mail: dusan.repovs@uni-lj.si

Institute for Mathematics, Physics and Mechanics, University of Ljubljana

e-mail: matija.cencelj@uni-lj.si
Поступило в редакцию 13 февраля 2003 г. 